\title{
Paternal Familial Twinning: Hypothesis and Genetic/Medical Implications
}

\author{
Michael Golubovsky \\ Division of Evolutionary Theory, Institute of the Science and Technology History, \\ Russian Academy of Science, St. Petersburg, Russia
}

\begin{abstract}
The phenomenon of paternally dependent familial twinning has been known in human and animal genetics since the 1920s, but still remains without any theoretical explanation and is indeed a neglected field of inquiry. Over the last two decades investigations in reproduction biology have discovered the significant role of multiple paternally dependent errors in fertilization including androgenic triploidy and moles. We suggest the hypothesis that the fathers of twins in the relevant families carry gene variants that increase the probability of dispermy, diplospermy and male pronucleus heterochrony as well as involvement of two male pronuclei in the fertilization of two female meiotic products. Any resulting twins would be an exceptional intermediate between MZ and DZ twins - and might properly be described as "sesquizygotic" (SZ). Paternal familial twinning may also go together with infertility due to triploidy, moles and chimerism. The hypothesis: (i) places the curiosities of paternally derived twinning within the framework of current knowledge of reproductive genetics and verifiable phenomena; (ii) predicts the existence of families in which twinning is associated with reproductive abnormalities; (iii) predicts an occurrence in relevant families of the third and intermediate category of SZ twins. Families with paternal twinning may thus provide the natural selective system for the search of unusual cases of primary chimeras, the frequency of which is still unknown.
\end{abstract}

Twinning may be viewed as a natural genetic engineering event, including cloning in the case of MZ twins. Comparison of DZ and MZ twins remains one of the most fruitful approaches in human genetics. However, many aspects of twinning are still unclear or need to be examined in light of current discoveries in reproductive genetics and cytology. These include (i) in human triploidy, the finding that an additional genome has paternal origin in about $75 \%$ cases; (ii) the deciphering of the androgenetic nature of partial and complete hydatidiform moles (HM); (iii) discovery of imprinting-dependent developmental differences between parental genomes; (iv) the recognition of the high primary frequency of twin zygotes, the phenomena of "vanishing twins" and confined placental chimerism (Boklage, 1995); (v) the finding that in the human zygote the centrosome regulating cleavage division is paternally inherited (see ref. to this point: Palermo et al., 1994; 1995; Sathananthan, 1998; Sutovsky \& Schatten, 2000).

The widespread use of in-vitro fertilization and embryo transfer procedures (IVF/ET) provides for the first time direct observations of the development pattern of oocytes, zygotes and early embryos. These studies significantly change a longstanding belief about precision associated with the basic reproductive processes in the human meiosis and gametogenesis, fertilization and early embryo development. All of them appear to be "remarkably imprecise" (Hassold, 1986) or error prone (Warburton, 1997). Simply stated, up to $35 \%$ of human oocytes have chromosomal abnormalities. Moreover, according to direct observations in assisted reproduction, up to $70 \%$ of embryos fail to implant. Only about $10 \%$ of transferred embryos produce full-term embryos (Zenzes \& Casper, 1992). An unusually high frequency of embryonal loss seems to be unique for humans in comparison with other mammals (Hassold, 1986). Further, cytogenetic and molecular studies in combination with direct IVF observations demonstrate hitherto totally unforeseen reproductive and developmental circumstances, for instance regular diploid androgenesis, in the case of complete hydatid moles (CHM), and different types of chimeras (Strain et al., 1998; Uehara et al., 1995; Verp et al., 1992), including extraordinary living parthenogenetic/normal chimeras (Giltay et al., 1998; Strain et al., 1995). Indeed it was even suggested that chimerism and parthenogenetic activation "may be relatively common in man with substantial developmental implications including brain and behaviour" (Surani, 1995).

\section{Multigenic Control of Reproduction and Twinning}

Cytogenetically an oocyte can be viewed in terms of a triple entity: a peculiar 2 n-gamete arrested at the MII stage, as a zygote after fertilization and as a pro-embryo containing the program of development. Female oogenesis is a unique process that includes two developmental blocks and continues over many years. Primary oocytes enter into meiosis during female embryonic development, attaining the diplotene stage around the time of female birth. Meiosis is then arrested for 12-15 years until the onset of puberty when growth and maturation of follicles occur. A few hours before an ovulation the first meiotic block is removed and the secondary oocyte with its first polar body becomes a distinct entity. At the MII stage a new meiotic block sets in.

Address for correspondence: 1812 Delaware str \# 205, Berkeley CA 94703, USA Email: innagol@uclink4.berkeley.edu or mdgolub@yahoo.com 
Only after sperm penetration is meiosis completed and two haploid products are formed. From the genetic point of view, it is important to emphasize that every human being starts its ontogenesis from a definite fertilized oocyte that in turn began to form as a germ cell during the embryogenesis of the individual's mother, or during the period of pregnancy of the grandmother. Thus, each specific egg cell both physically and genetically links a total of three generations! From this perspective, twinning occurrence must be analyzed in connection with the deviations in all three multigenic processes: meiosis and gametogenesis, fertilization and the early stage of embryogenesis.

A series of distinct genes may induce twinning in humans as an abnormal variant of fertilization and development. Fertilization is a genetically controlled multistep process that includes three main stages: 1) sperm entry, 2) pronuclei formation and 3) first mitosis of male and female pronuclei (Fitch et al., 1998). Sperm entry results in completion of female meiosis blocked after ovulation at the MII stage, sperm plasma membrane and nuclear envelope breakdown, centrosome separation and replication. The second stage (pronucleii formation) includes sperm decondensation and nuclear envelope reformation, chromosome protein exchange (signaling) between male and female pronuclei, pronuclear migration, their apposition and synchronization of DNA replication. The third and final stage includes mitotic spindle formation, chromatin condensation and initiation of mitotic cycle. Mutations disrupting some of the genes controlling any of these steps may lead to abnormal fertilization and twinning.

The initial findings of mammalian reproductive candidate genes confirm the intricate complexity of genetic and developmental events related with twinning. FOXL2 is the human gene required for the maintenance of ovarian follicles. It is a member of helix/forked family of transcription factors mapped in the region of 3q23. Haplo-insufficiency of this gene (heterozygosity on deletion) is connected with eyelid defect and premature ovarian failure syndrome (Pruett \& Zinn, 2001). In contrast, a further mammalian candidate gene, BMP15 mutated in sheep carrying the $\mathrm{FecX}$ locus, induces multiple ovulation and twinning. It has a peculiar dominant dosage-dependent expression. Heterozygous females $(\mathrm{FecX} /+)$ have increased ovulation rate and twinning, but mutant homozygotes $(\mathrm{FecX} / \mathrm{FecX})$ express primary ovarian failure and are sterile (Galloway et al., 2001). BMP15 maps to human chromosome

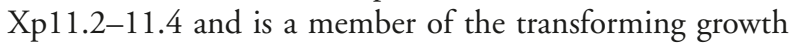
factor TGF family, specifically expressed in oocytes. A mutation in the bone morphogenetic protein receptor BMPR1B also increases ovulation rate and twinning in sheep (Wilson et al., 2001), but there was no evidence of linkage to DZ twinning with markers from the synthenic region of human chromosome 4 containing BMPR1B (Duffy et al., 2001).

Finally, an analysis of one unique Lebanese family with recurrent hydatidiform moles of biparental origin not only permits understanding of the relevant autosomal recessive gene inducing hydatid mole formation mechanism, but also adds new insights into the molecular genetics of early embryogenesis and parental imprinting regulation
(Mogalbey et al., 1999). Similar convincing lessons of the value of the familial analysis are provided by the longterm studies of sex determination problems - especially the discovery of families with XX males and hermaphrodites not having SRY DNA (McElreavey \& Fellous, 1999; Oster, 2000).

\section{Conceptual Controversies and Background of the Problem.}

Many controversies exist regarding the origin and developmental genetics of MZ twins (Hall, 1996; Machin, 1996; Machin \& Keith, 1999). An elegant molecular approach to the comparison of $\mathrm{X}$-inactivation profiles among three placental and fetal membrane groups of $\mathrm{MZ}$ twins confirmed the difference between them in the timing of the twinning event. It was found that X-inactivation precedes the twinning event in the predominant subgroup of monochorionic MZ twins and occurs when there are -10 cells in the embryo before the blastocyst stage (Chitnis et al., 1999; Monteiro et al., 1998). These studies show that Xinactivation does not play a direct role in the twinning event and this decision occurs at the level of the embryonic cell population.

Despite these findings and the observation of thousands of microsurgical manipulations during IVF and ICSI procedures "the de-novo embryonic fission apparently required for spontaneous $\mathrm{MZ}$ twinning has never been directly observed" (Sills, 2000). This means that the "splitting" mechanism remains somewhat a metaphor in spite of recent experimental clonal propagation of primate offspring by artificial reaggregation of blastomeres of eight-cell embryo (Chan et al., 2000). Analogous is that long period when the term "splitting" was used as a description of chromosome behavior in meiosis. It turned out to be just a metaphor. There is not in fact any splitting, but replication of chromosomes and segregation. Even the term "fusion" as a description of fertilization behavior of male and female pronuclei is somewhat a metaphor, due to recently established substantial topological genome separation of two parental chromosome sets and distinct patterns of their methylation-demethylation during first two cleavage mitoses. The nuclear compartmentalization of mammalian parental genomes was seen even in most nuclei of the four cell embryos (Haaf et al., 2001).

As Boklage (1987) so aptly noted, the classical research heuristic is "so deeply entrenched that few give it a thought". He then put forth a stimulating but unorthodox concept that MZ and some part of DZ twinning is somehow related to symmetry determination in the oocyte. He also suggested what was a reaffirmation of a previously hypothesized possibility of so called "tertiary oocytes" with a symmetrical second meiotic division yielding two potential gametes from a single oocyte. This possibility seems consistent with the direct cytological observations of $5-18 \%$ of activated two-pronuclear oocytes without extrusion of second polar body (Taylor et al., 1994; Zenzes \& Casper, 1992).

An essential part of the symmetry concept is based on the facts of familial association of both MZ and DZ twinning with an excess of lefthandedness and malformations 
resulting from the fusion of bilateral embryonic halves. These familial features are explained by the hereditary transmission of a factor which weakens the oocyte and proembryo symmetry determination (Boklage, 1987). The recent conclusion that the specification of the anterio-posterio axis and relevant asymmetries in mammals depends on information that already present in the zygote before it embarks on cleavage (Gardner, 2001) supports this concept. Some genealogical data also indicates such an association in families of MZ as well as DZ twins (Michels \& Ricardi, 1981).

The present paper draws attention to yet another controversial question in twinning genetics; that is, the occurrence of paternally derived familial twinning. As early as the 1920s Davenport, an early American geneticist, presented statistical data arguing that twinning frequency may depend not only on the maternal but also on the paternal line. He suggested that gametes of certain males can contribute some factors which increase the viability of twin zygotes resulting from multiple ovulation. Since this publication, additional observations in animals and humans discuss paternal-derived familial twinning (for review of old references see: Bomsel-Helmreich \& AlMufti, 1995; Bulmer, 1970; Kanaev, 1959; Parisi et al., 1983).

Recently St Clair found a unique cluster of families with a paternal influence on twinning after an examination of all Scottish records for the period 1800-2000 years with one particular surname. In total, 50,000 births were examined (St Clair \& Golubovsky, 2002). The detailed genetic and molecular analysis of specific familial cases may strongly contribute to reproductive genetic phenomena and has important medical and scientific implications (St Clair et al., 1998).

The task of this paper is to hypothesize what specific genetic factors are transmitted from the fathers to male gametes, when these interact with the oocyte, and which of these putative factors result in more then one zygote and embryo. Variation in gamete genetics is suggested as the explanation for the "fathers and twins" enigma. The proposed hypothesis comprises findings lying on the border of three basic reproductive processes: meiosis and gametogenesis, fertilization and early embryogenesis. Suggested is the existence of definite paternal genes, which are expressed in male gametes and lead to dispermy or to heterochronous mitotic division of male pronucleus. The third source might be a male meiotic mutation, which blocks male meiosis and increases the fraction of diploid gametes (diplospermy) in the paternal gametic pool (Golubovsky \& Golubovskaya, 1984). The final and common outcome of all these events might be an appearance of two male pronuclei in the egg with subsequent double fertilization of two female meiotic products.

According to this hypothesis, the birth of paternaldependent twins appears to be a fortunate result of deviant fertilization scenarios that result mostly in miscarriages and partial infertility exhibiting itself as diandric triploidy and diploid androgenesis (complete HD). It is also possible to expect in relevant families the Inferility/Twinning Paternally Dependent or ITPD syndrome (Golubovsky, 1998). There are two additional important implications of the hypothesis
- first, the appearance in such families of sesquizygotic (SZ) twins intermediate between MZ and DZ twins, and second, the appearance of chimeras both among twins and their siblings. The current data on reproductive genetics are consistent with the premises of the idea.

\section{Genetic Aspects of Familial Twinning}

The pedigrees of familial twins may be roughly subdivided into three main groups: those with predominant $\mathrm{DZ}$ twinning, those in which $M Z$ twins predominate, and finally families with tendency to produce $\mathrm{DZ}$ as well as $\mathrm{MZ}$ twins. When DZ twinning was modelled as a parity trait, the data were compatible with an autosomal dominant gene with a population frequency of 0.3 and low penetrance for female gene carriers (Meulemans et al., 1996). Keeping in mind the multigenic control of gametogenesis as well as fertilization, it is apparent that families with DZ twin inheritance must be genetically heterogenous. This simple prediction is in agreement with the recent report of a family with DZ female twin births in three successive generations (Andreano et al., 1998).

Specific families with clear MZ twin inheritance are also described (Harvey et al., 1977; Shapiro et al., 1981) and a detailed description of a series of such families was recently reported (Machin \& Keith, 1999). These pedigrees show a dominant gene with variable penetrance and expression. Curiously, inheritance of familial MZ twinning was observed both through maternal and direct paternal influences (Harvey et al., 1977). A recent population study demonstrated an increased risk for monozygotic twin mothers to have monozygotic twin offspring (Lichtenstein et al., 1998). In contrast, a paternal effect on $M Z$ twinning was not demonstrated. The authors concluded that the gene(s) increasing the tendency of the embryo to split are expressed in the mother but not in the fertilized egg.

It is usually believed that the genetic components of $\mathrm{MZ}$ and $\mathrm{DZ}$ twinning are independent. However the results of a comprehensive study of familial twinning demonstrated that in some families, the same heritable factor(s) might predispose both to MZ and DZ twinning. In other words, heritability might involve both the maternal and paternal line (Michels \& Ricardi, 1981). Other data also indicate an intrinsic embryo-genetic tendency to produce both $\mathrm{DZ}$ and $\mathrm{MZ}$ twins. Thus, among 15 sets of triplets conceived spontaneously, six sets were monozygotic, 7 were dizygotic and only 2 sets were trizygotic (Machin \& Bamforth, 1996).

In an attempt to produce a theoretical explanation of this tendency, it was suggested that the chemical modulation of cell-adhesion molecules (CAM) regulating cell movements and the topological development of tissues might account for the hereditary influence on both $M Z$ and DZ twinning. The MZ inherited induction might be due to a premature onset of the specific CAM that reduces cell adhesion between first blastomeres. The ontogenetic disregulations that occur when organisms change the relative times of appearance and the rates of development for specific characters are characterized as "heterochrony" (Gilbert, 1998). 
Heterochronic events are important phenomena in developmental biology and evolution. Premature reduced cell adhesion might occur at the time when the post-zygotic embryo cells are still totipotent. In normal conditions the CAM mediated weakening of cell contacts begins at later stages of cleavage. At the same time, mutant CAM action at the level of follicles may result in simultaneous production of two or more follicles (Gedda \& Brenchi, 1986). The authors illustrated this possibility by describing an impressive case of sextuplets born in 1985 in Italy. The six infants appeared to result from distinct fertilisation of three ovulated eggs, two of which produced $M Z$ twin derivatives (two MZ cotwins with single chorion and two amnions and three $M Z$ cotwins with single chorion and three amnions). The first such inner cell mass-specific cell adhesion molecular was identified in the mouse blastocyst. It is located at cell-cell borders and expressed at the very first signs of blastocoel formation (Robson et al., 2001).

\section{Fathers and Twins: Familial Data}

In some genealogical records, the paternal influence on twinning exhibits a rather stable hereditary character. For instance, among the 1333 descendants of Jean Bertholet who emigrated from France to Pennsylvania in 1726, 22 sets of twins or 3.3\% were observed in a period of 7 generations (Detwiler, 1933). A high proportion of kinships with a parternal contribution to twinning was also reported for Utah Mormon genealogies (Carmelli et al., 1981). In addition, Michels and Ricardi (1981) found a direct paternal influence in two Milwaukee (Wisconsin, USA) twin groups. Among 32 families with three or more twin sets, there were 8 instances $(25 \%)$ in which a male was a critical genetic intermediary between related twin sets. Both $\mathrm{MZ}$ and DZ twins were found. Some families demonstrated both direct paternal influence and transmission of relevant factor from male ancestors to woman giving birth to twins (Michels \& Ricardi, 1981).

\section{DISPERMY}

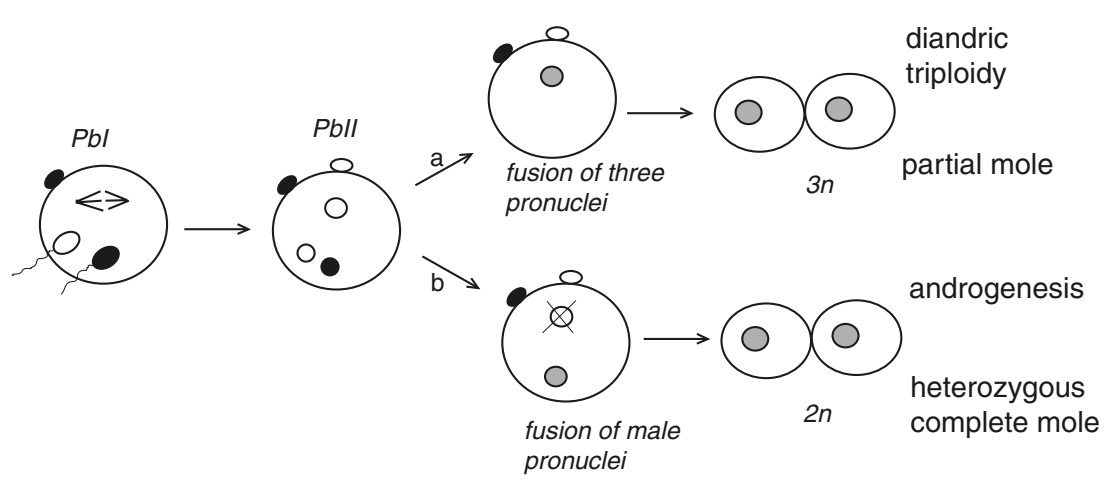

\section{HETEROCHRONY}

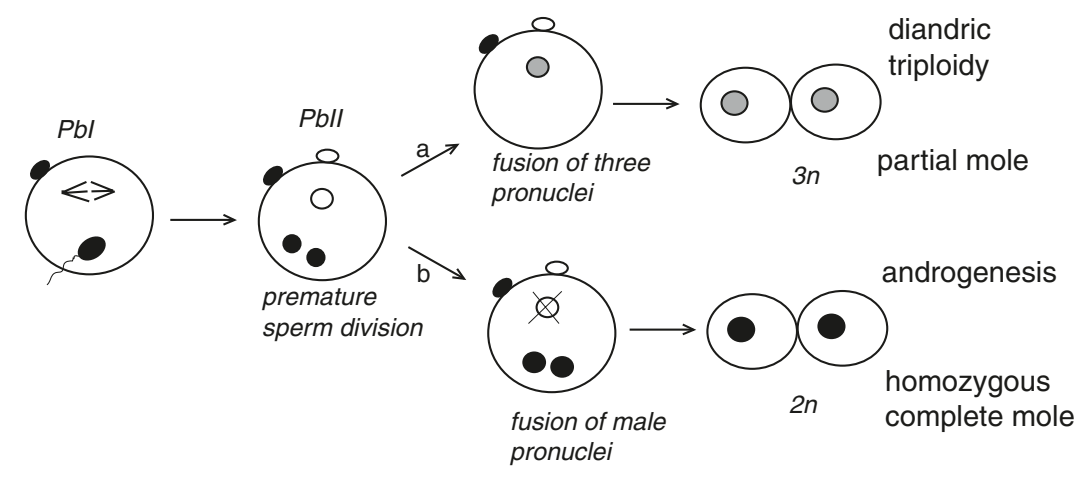

\section{Figure 1}

Two paternal-dependent variants of abnormal fertilization leading to triploidy and hydatidiform moles.

I. Dispermy with variants: (a) Diandric triploidy associated with partial mole; (b) Diploid androgenesis resulting in heterozygous complete mole. II. Heterochrony, with formation two male pronuclei followed by (a) Diandric triploidy or (b) Diploid androgenesis leading to homozygous hydatidiform mole.

$\mathrm{Pb} 1$ and $\mathrm{Pb}$ II designate first and second polar bodies. 
A similar study based on analysis of the London twin population reports that among 10 families with familial monozygotic twinning direct paternal influence or transmission through the male via a dominant MZ-inducing factor was expressed in three families (Harvey et al., 1977).

\section{$\overline{\text { Fertilization Scenarios with Erroneous }}$ Paternal Involvement}

There are two cytogenetic deviations where paternally dependent errors occur regularly and with rather high frequency - dispermy and heterochrony. These in turn lead to significant reproductive abnormalities such as triploidy and hydatidiform mole (Figure 1). A reasonable estimate suggests that overall $7 \%$ of human oocytes are penetrated by more than one spermatozoon (Feng \& Gordon, 1996). Triploidy in humans occurs in near $1 \%$ of all clinically recognizable pregnancies. Most triploids die early in development and they constitute nearly $10 \%$ of all spontaneous abortions. It is now firmly established that an additional genome in triploids has a paternal origin in nearly 2/3 of cases (Uchida \& Freeman, 1985).

Recent comprehensive molecular analyses based on selected microsatellite markers located near and distal to the centromeres discriminates between six possibilities for the origin of triploidy: failure of meiosis I; failure of meiosis II; fertilization by two gametes (dispermy or "dieggy"); involvement of diploid gametes; non-meiotic germ cell; and postmeiotic cell errors (Zaragoza et al., 2000). It was confirmed that in about $70 \%$ of cases, the triploidy had diandric origin. Among the diandric triploids, $86 \%$ were connected with dispermy, others resulted from male meiotic errors. In either case, penetration of two sperm indicates a failure of the polyspermy block.

The penetration process is presently regarded as a multistep genetically controlled process involving the so-called molecular dialogue between glycoproteins of the zona pellucida and protein receptors on the sperm head. Penetration itself includes at least 5 main molecular events (Wasserman et al., 2001). Mutations in maternal and paternal genes could lead to failure in the polyspermy block.

Phenotypic effects of digynic and diandric triploidy are quite different. In $-50 \%$ of cases diandric triploidy leads to so called partial hydatidiform mole (HM) whereby the human conceptus displays macroscopically visible vesicular villi, in combination with trophoblast hyperplasia and retardation of fetal development. On the other hand, in complete hydatidiform moles (CHM) the fetus and amniotic membranes are usually absent, and the conceptus transforms itself into a grape-like structure connected with gestational trophoblastic disease or chorioncarcinoma. HM occurs in 1 in every 1500 pregnancies in the USA. The incidence of HMs is 5 to 15 times higher in eastern Asia and Japan. Between 35 and 39 years of age the relative risk of $\mathrm{HM}$ is $2.5 \%$, reaching $9.8 \%$ after age of 40 (Lindor et al., 1992).

The striking cytogenetic discovery that complete HM are androgenic diploids was made at the end of the 1970 s. It is estimated that about $20 \%$ of complete HMs carry two paternal genomes due to expected dispermy. This presumably results from fusion of two male haploid pronuclei with an elimination of maternal pronucleus. Surprisingly, more than $70 \%$ of dipolid CHMs appeared to be homozygous, either occurring from fertilization of an anuclear egg by a haploid sperm with subsequent endoreplication, or due to heterochrony; that is, the premature mitotic division of one male haploid pronucleus with subsequent fusion (diploidisation) of these two mitotic products and disappearance of the egg pronucleus (Figure $1 \mathrm{~b}$ ).

In other words, spontaneous natural androgenesis occurs rather regularly in humans. Diploid CHM are more aggressive than partial ones. It is not clear why the female pronucleus is eliminated. One potential explanation is that the presence of an additional male pronucleus drastically distorts both compartmentalization and some vulnerable gene dosage dependent interactions during the so-called molecular dialogue between male and female pronuclei. This phenomenon may stop ontogenesis in the female pronucleus and possibly induce zygotic ontogenetic instability. Similar dispermy attributable "generalized unbalanced mosaicism" has been described (Zuelzer et al., 1964). It should be noted that vulnerable parental-dependent gene dosage regulation constitutes the basis and essence of the imprinting process.

\section{$\overline{\text { Multiple Developmental Zygote Instability }}$ and Chimeras}

New methods of molecular diagnostics significantly extend our understanding of possible errors that may occur during fertilization and early development. In the context of this paper, three thoroughly analyzed findings of unusual chimeras in humans are depicted. Figure 2 a presents a parthenogenetic/normal chimera (Giltay et al., 1998; Strain et al., 1995); Figure $2 \mathrm{~b}$ presents a chimera comprising two paternal and one maternal genome (Surani, 1995) and Figure 2c, a tetragametic aggregation chimera (Strain et al., 1995; Uehara et al., 1995).

In the first cases a phenotypic boy with facial asymmetry and small testes was presented. His blood appeared to be XX with maternal disomy on both $\mathrm{X}$ chromosomes and autosomes. At the same time, his fibroblasts demonstrated biparental codominant inheritance with the same maternal genome as in XX-blood cells.

Three possible mechanisms for the origin of such chimeras with maternal disomy in two cell populations were considered; all include heterochronic events (Surani, 1995). In the first, fertilization is followed by an equal cytoplasmic egg division with an extrusion of a large second polar body which then develops as the gynogenetic blastomere undergoing diploidisation. Subsequently, the two cell lines mix or aggregate and a gynogenetic/normal chimera occurs. In the second mechanism, parthenogenetic activation of the oocyte is followed by immediate cleavage producing two haploid parthenogenetic blastomeres. One of them is fertilized while the other becomes diploid resulting in a normal/parthenogenetic chimera. Both of these possibilities did not account for the observed isodisomy (identity) for all maternal chromosomes and the evidence from distal microsatellite markers (due to recombination at MI of meiosis), the haploid products of the second meiotic division must not be identical). In the final mechanism, 
a

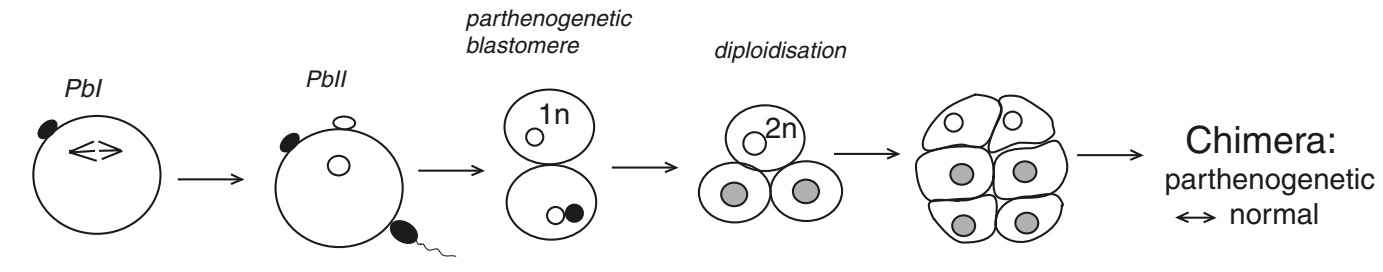

activation before fertilization

b

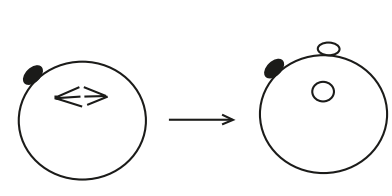

activation \& cleavage before fertilization

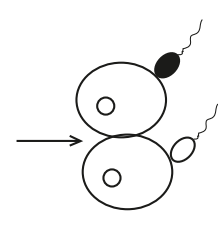

double fertilization

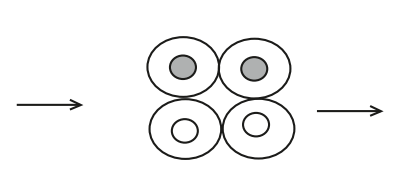

blastomere fusion
Chimera:

two paternal

one maternal

genomes

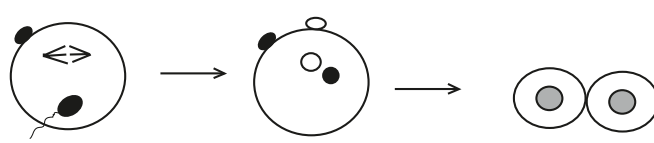

C

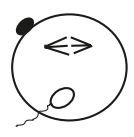

fertilization

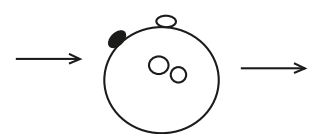

two zygotes
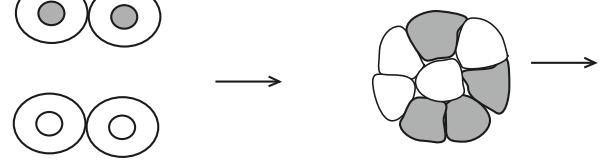

Chimera:

tetragametic

\section{Figure 2}

Variants of the origin of some unusual chimera discovered in humans.

a) Heterochrony resulting in zygote instability and simultaneous combination of some rare developmental events: Egg activation and extrusion of Pbll occurs before fertilization; Sperm nucleus enters in mitotic cycle in one blastomere; the second blastomere undergoes diploidisation and diploid parthenogenetic/normal chimera appears having identical maternal genome in two cell lineages (modified on Strain et al., 1995; Surani, 1995);

b) Heterochrony in combination with double fertilization result in chimera with two Paternal and identical maternal genome (modified on Giltay et al., 1998):

c) Tetragametic chimera appearing due to aggregation of two zygotes (modified on Verp et al., 1992; Uehara et al., 1995; Strain et al., 1995).

fertilization occurs after an oocyte activation and after PbII extrusion. Two haploid blastomeres appear, the sperm nucleus enters the mitotic cycle in one of the blastomeres, whereas the second haploid blastomere develops parthenogenetically, becomes diploid and a parthenogenetic/normal chimeras occur. The last eventuality as depicted in Figure 2a corresponded to all genetic data.

Certain conclusions may be deduced from this remarkable finding. First, it shows the vital importance of reproductive heterochrony. Second, it represents an example of the simultaneous implementation of a series of rare, but deviant reproductive events: egg parthenogenetic activation, the possibility of sperm penetration in the blastomere, delayed sperm entry in mitotic division, early diploidisation of one blastomere, constitution of an aggregated normal/parthenogenetic chimera, and survival of this chimera. One may thus conclude that heterochrony induces zygotic ontogenetic instability in which multiple developmental abnormalities are possible.

Before this chimera was described most reproductive biologists and experienced clinicians would have thought it unbelievable. Surani (1995) concluded that spontaneous parthenogenetic activation and ontogenesis may be relatively common in man, but remain undetected due to disbalance and early abortions. Such chimeras may have peculiar phenotypic deviations. For instance the authors noted that left sided microsomia of the chimeric boy may have resulted from retarded growth of tissues with parthenogenetic cell lineage (Strain et al., 1995). Interestingly, in similar mouse chimeras, parthenogenetic cells contribute to brain in higher numbers that to other tissues.

Figure $2 \mathrm{~b}$ illustrates the origin of an unusual type of chimerism in a 5 year old proband with true hermaphroditism and 46,XX/46,XY karyotype (ratio 38:12). On the 
physical examination, he exhibited a normal phallus, normal right testis, but bisexual left gonad. In all other respects he was developing normally. Molecular analysis showed double paternal and single maternal genetic contribution. A possible explanation also includes heterochrony: a premature activation and immediate ovum cleavage, followed by double fertilization of two blastomeres followed by subsequent blastomere aggregation.

Figure 2c shows a variant of origin of tetragametic chimeras with double paternal and double maternal genomes. Theoretically, tetragametic diploid chimeras may occur as the result of several mechanisms: fertilization of the ovum and first polar body, fertilization of the ovum and second polar body, and via mixing or amalgamation of independently ovulated and separately fertilized eggs. The last outcome, observed by some authors, is depicted on Figure 2c. The phenotypes of unusual probands were as follows: true hermaphrodite $46, \mathrm{XX} / 46, \mathrm{XY}$ with a ratio of two cell lineages 18:7 (Verp et al., 1992).

A hermaphroditic boy conceived by in vitro fertilization was described with a constitution of $46, \mathrm{XX} / 46, \mathrm{XY}$ and varying proportions of XY and XX cells in each culture (Strain et al., 1998). Interestingly, the phenotypes of $\mathrm{XX} / \mathrm{XY}$ chimeras vary from normal fertile males through males with ambiguous genitalia and female fertile hermaphrodite to phenotypically normal females. In contrast, same-sexed chimeras should be normal and mostly unnoticed. The discovery of chimerism needs special types of molecular genetic analysis and it is not known how often primary chimeras occur either spontaneously or after assisted reproduction.

\section{Cytogenetic Possibilites of Paternaly-derived Twinning}

The origin of triploidy and complete HM showed significant involvement of two paternally derived events dispermy and heterochrony (Figure 1). Analysis of chimeras made clear that the heterochrony occurring during the fertilization process results in zygotic ontogenetic instability and multiple unusual variants of development (Figure 2). The same events may also lead to the occurrence of twins.

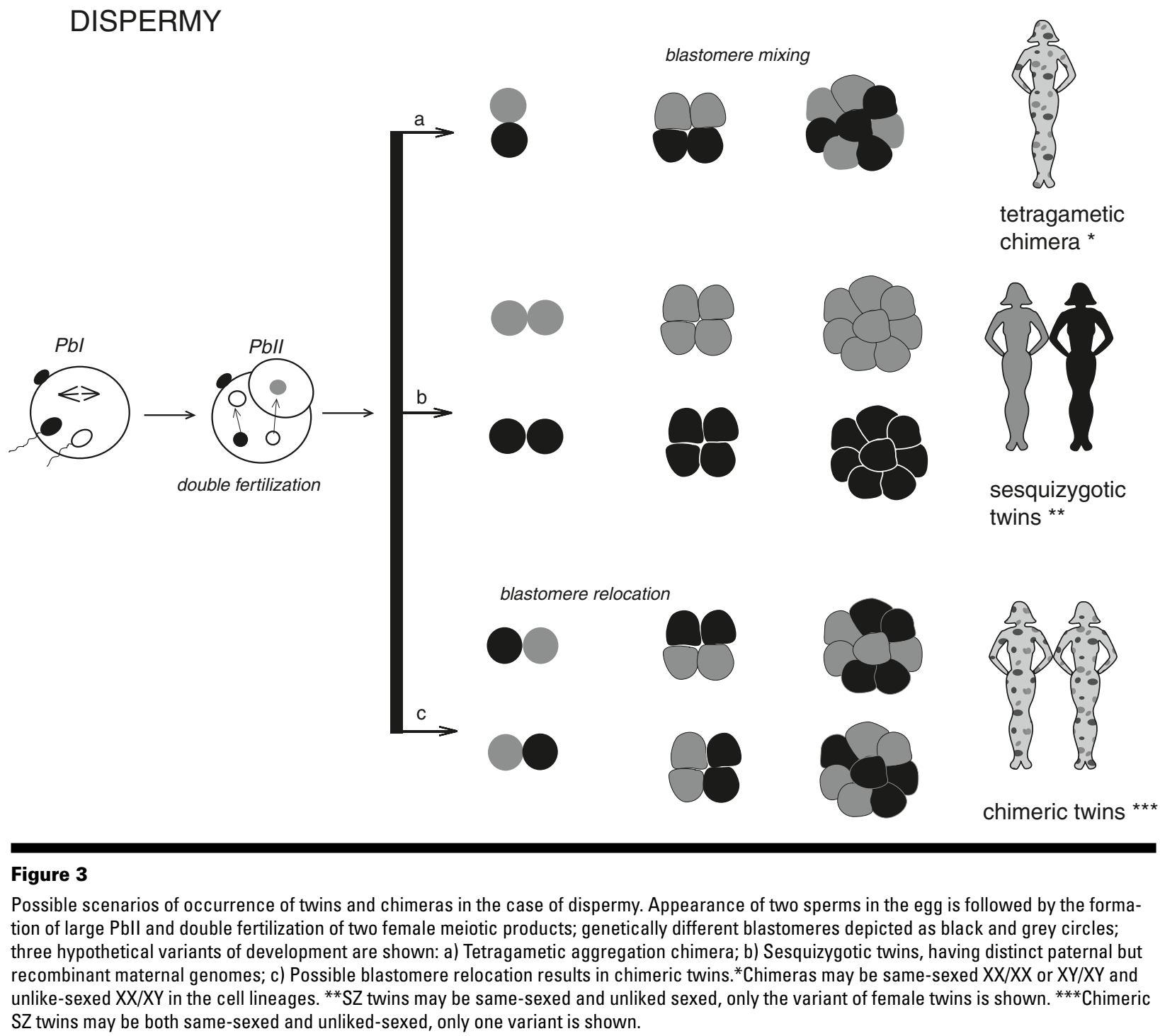

Twin Research April 2002 
In the case of dispermy, two male pronuclei usually fuse with the female pronucleus and produce triploids, or they fuse with each other. In this instance, the female pronucleus is eliminated and a complete heterozygous HM occurs (Figure 1a). We suggest an additional scenario, whereby dispermy, followed by equal oocyte division leads to double fertilization. We hypothesize three possible variations of double fertilization (Figure 3): (a) blastomere aggregation and occurrence of tetragametic chimera; (b) independent development of two blastomeres and occurence of twins characterized "sesquizygotic"; and (c) the appearance of chimeric twins due to assumed blastomere relocation. Viable tetragametic chimeras may exhibit a constitution of XX/XX or XX/XY. Dispermic sesquizygotic or SZ twins may be either like-sexed or unlike-sexed. In contrast dispermic chimeric twins must be the same sex. However, in the case of the XX/XY constitution, the twins may be discordant for phenotypic sex as observed for similar types of chimeras or mosaics (for reference see Strain et al., 1998).

\section{HETEROCHRONY}

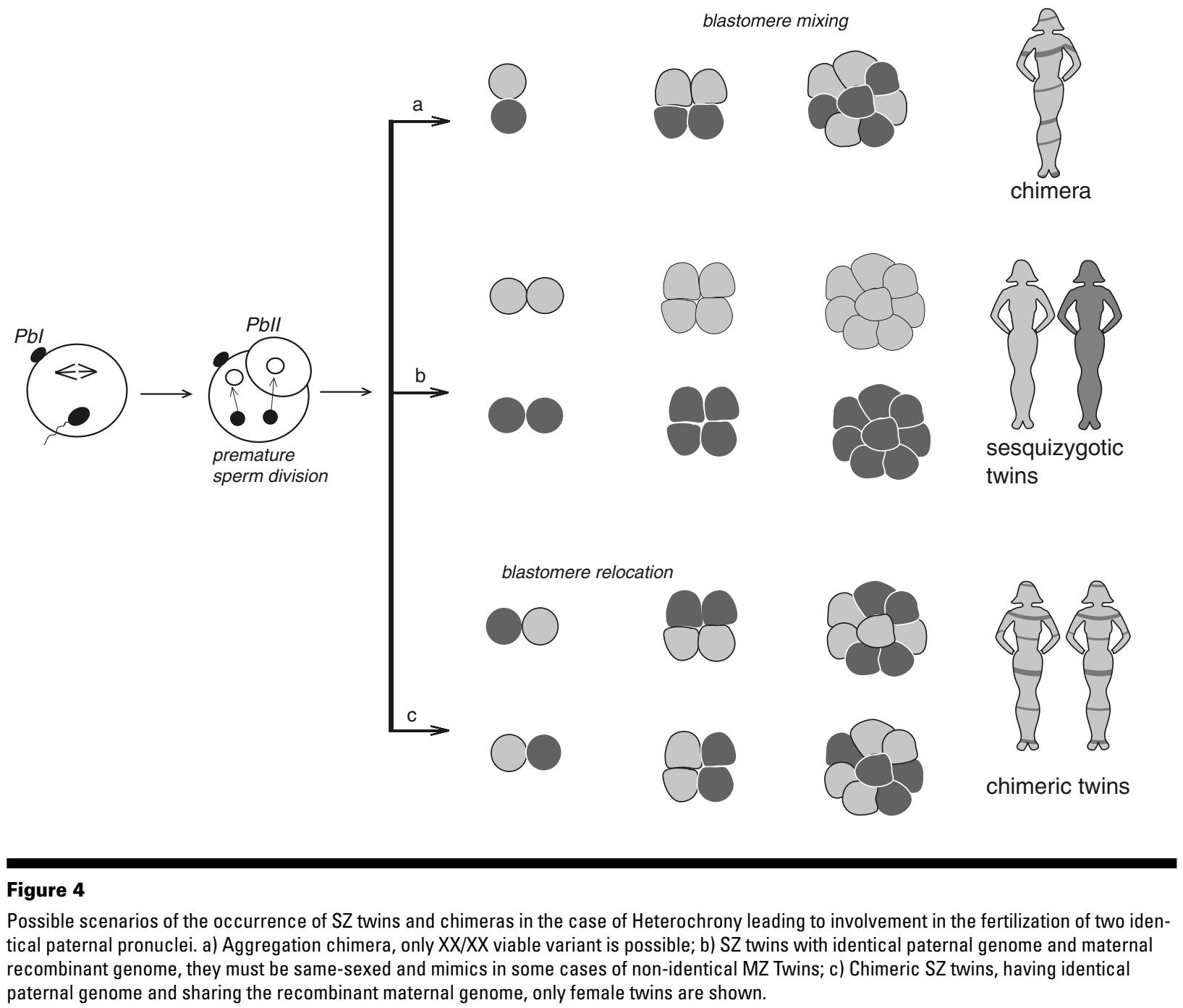

Dispermic SZ twins in principle resemble normal DZ twins, but are more similar in their maternal genome. In brief, two layers or levels of genetic variability are implemented through two meiotic divisions, the segregational and recombinational ones. The first division provides the Mendelian independent segregation of the different pairs of homologous chromosomes with an expected number of variants $2^{n}$ where " $n$ " is the number of chromosome pairs. Crossing over or recombination between homologous chromosomes in MI prophase constitutes the other level of genetic variability. The segregation-derived variability occurs even when recombination is supressed as is the case in the males of Drosophila or in the pericentromeric regions of chromosomes or in small acrocentric chromosomes. The ratio between segregational and recombination variabilty is the function of the number of chromosomes and their length (Goldar \& Kimberling, 1981).

For humans with 23 chromosome pairs the main potential for variability occurs through the regular chromosome segregation at the first meiotic division. Thus, 
maternal products of the second meiotic division are expected to be identical if we exclude homologous recombination. This is the reason why the maternal genomic component in dispermic SZ twins is expected to be more similar than in true DZ twins. Based on the other approach, Boklage (1987) also made an assumption that some of DZ twins may be in reality "polar body DZ twins," or using my terminology, SZ twins. Current microsatellite markers with selection of both pericentromeric and distant markers allows the discrimination between real DZ and SZ twins, but this is a task for a future studies.

The same three fertilization outcomes are possible if two male pronuclei are present as a result of premature male pronucleus mitotic division or heterochrony rather than from dispermy (Figure 4). However, in all such cases (chimera, sesquizygotic and chimeric twins) the paternal contribution is expected to be identical. Thus heterochronous $S Z$ twins having identical paternal genomes are expected to be like-sexed and differ only by recombination events in maternal chromosomes. Phenotypically they will look like MZ twins being non-identical on some maternal heteroallelic genetic loci. Chimeric heterochronous SZ twins will also be like-sexed and appear as dissimilar or non-identical $\mathrm{MZ}$ twins.

A third possible mechanism may lead to the appearance of two male pronuclei in the ovum. This consists of male meiotic mutation like diplospermy, which blocks meiosis at the MI or MII stage. Participation of $2 \mathrm{n}$ male gametes in fertilization explains more than $13 \%$ of cases of diandric triploids (Zaragoza et al., 2000). The mean incidence of diplospermy is about $0.1 \%$ in healthy, fertile males. However, among 45 infertile men two patients exhibited significally increased diplospermy rates of 0.35 and 1.6\% (Guttenbach et al., 1997). The initial description of mammallian diplospermy meiotic mutation was reported in the semen of a golden retriever. Over a period of two years the 3 -year male produced from to $37-60 \%$ of giant head diploid sperm. However, the dog produced a litter of healthy puppies (Dahlbom et al., 1997).

The immature spermatocytes blocked at MI or MII stage of meiosis as shown in remarkable cyto/embryological experiments can undergo meiotic divisions in maturing oocytes and participate in the formation of diploid embryos and living progeny (Oguza et al., 1997, 1998; Sasagawa et al., 1998). These findings provided direct evidence that the nuclei of male germ cells acquire the ability to fertilize oocytes before the first meiotic division. Such male gametes completing meiosis in the ovum may produce two haploid pronuclei (initially suggested in Golubovsky \& Golubovskaya, 1984) and subsequently participate in fertilization as depicted in Figures 3 and 4. In the cases of MII block, same-sexed SZ twins are expected. They will have the same centromere markers in both genomes but differ at informative distal alleles. Under these circumstances, from a genotypic point of view, $S Z$ twins may represent the whole spectrum of intermediates between true $\mathrm{MZ}$ and $\mathrm{DZ}$ twins.

This theoretical prediction provides an explanation for doubtful cases of zygosity observed regularly during mass zygotic screening. For example, among 292 pairs of twins described by Ardashnikov et al. (1936) who were thoroughly diagnosed on 15 phenotypic characters, 137 pairs were clearly classified as monozygotic and 148 as dizygotic. However seven pairs or $2 \%$ could not be placed in either category. During mass screening in other studies researchers find themselves confronted with similar situations, especially in the cases of dissimilar MZ twins (Machin, 1996; St Clair et al., 1998).

Families with paternal hereditary twinning may be heterogenous due to the possibile action of (at least) three distinct cytogenetic events - dispermy, diplospermy and heterochrony. In the case of diplospermy and heterochrony one may expect a familial prevalence of same-sexed twins and these assumed $\mathrm{SZ}$ twins may pass as MZ twins. There are numerous cases of discordance between $\mathrm{MZ}$ twins in phenotype and/or genotype (Machin, 1996) as recently summarized in a special monograph (Machin \& Keith, 1999). The sources and limits of MZ dissimilarity (nonidentity) have important medical and social implications (St Clair et al., 1998). The main genetic and epigenetic factors inducing $\mathrm{MZ}$ discordance are differential imprinting, skewed X-inactivation, post-zygotic gene and chromosome mutations.

In addition, the suggested existence of heterochronous SZ twins and chimeras (Figure 4) makes the problem of $M Z$ twin discordance even more complicated. Indeed, some discordant MZ twins may in reality look like SZ twins and vice versa. One needs to have molecular genotype data in both parents and twins to discriminate among the possibilities.

\section{Verification and Implications of the Hypothesis}

It is useful to summarize the main premises and indirect evidence underlying the paternal-twinning hypothesis. First, reliable familial observations obtained since 1920s, both in human and animal genetics, describe the paternal component in inherited twinning. However, this phenomenon remained without any theoretical explanation for many years and was treated as unbelievable, doubtful, or marginal. Now however, sufficient evidence has accumulated to suggest this concept merits further consideration.

In particular, in two last decades, the significant role of paternally-dependent events in reproductive abnormalities has been described. These comprise dispermy, diplospermy and pronucleus heterochrony resulting in diandric triploidy and diploid androgenesis (complete HM). The regular appearance of two male pronuclei, due to distinct paternally derived events, makes possible an additional associated scenario: Involvement of two male pronuclei in fertilization of two meiotic products of oogenesis. Thus the birth of some twins can be viewed as a fortunate outcome of more frequent malformations connected with triploidy and diploid androgenesis, and confirms the clinical adage that twins are adaptive forms of congenital malformations.

The hypothesis predicts that pedigrees with paternally derived twinning may also express various reproductive abnormalities including infertility. Indeed, there are direct cytogenetic data about familial associations of triploidy with twinning (Uchida \& Freeman, 1985) and triploidy 
in-vitro with recurrent HM from a different partner (Pal et al., 1996).

The third premise of the hypothesis is the phenomenon characterised as zygotic ontogenetic instability. It is deduced from the discovery of extraordinary human chimeras resulting from the simultaneous implementation of a series of rare pre- and post fertilization events. Zygotic instability is frequently induced by the heterochrony of male and female cells during fertilization process (Figure 3). As one of its outcomes paternally dependent heterochrony may lead to $S Z$ twins and chimeras.

The validity of the hypothesis could be verified in two ways. The first would consist of a systematic search for and a detailed reproductive description of families with inherited paternally-derived twinning. Preliminary observations are positive on heterogeneity and on the character of transmission and expression of hypothetical paternal twinning factor(s). The recent discovery of the cluster of such families in Scotland (St Clair \& Golubovsky, 2002) merits thorough genetic analysis using current molecular techniques.

The second way in which the hypothesis can be verified is by means of special cytologenetic and molecular analysis of so called doubtful cases of twinning. Doubtful twins that classified as dissimilar MZ may be sesquizygotic or chimeric twins.

The hypothesis developed has some important predictions: It suggests an existence of definite reproductive entity: the Infertility/Twinning Paternally Dependent (ITPD) syndrome (Golubovsky, 1998). From this point of view, it will be important to have more detailed data about reproductive patterns among twinning families. Unfortunately, Twin Registers as a rule do not contain such data even for mothers of twins. We described one such family where hereditary twinning was associated with hereditary infertility (Golubovsky, 1984). Finally, the Old Testament describes an association between paternally derived twinning and infertility in the famous genealogy of Abraham (Golubovsky, 1986). Biblical authors present such detailed descriptions of human reproduction patterns (female and male infertility, post-menopausal conception, the course of pregnancy, the art of midwifery) that modern obstetricians and gynecologists can analyze specific cases of twining delivery and zygosity (Blickstein \& Gurewitch, 1998).

The hypothesis also provides an additional approach to the complex problem of the discordance of MZ twins. Even the Keith twins, Louis and his brother Donald, founders of the Center for the Study of Multiple Births, erroneously believed for many years that they were DZ twins, because they were dichorionic and not identical for some phenotypic traits (see the detailed description in the monograph of Machin \& Keith, 1999). Later blood typing and fingerprint analysis confirmed a high probability (99.6\%) of $\mathrm{MZ}$ status. Later analysis of microsatellite markers showed clear allelic differences at one locus (Keith \& Machin, 1997) even though DNA RFLP analysis was consistent with MZ status. The Keith twins inclined to explain this molecular "clinical paradox" as the result of postzygotic mutation. However this conclusion seems premature, as it cannot be excluded that the Keith twins are sesquizygotic and pass as dissimilar MZ twins. Only two-generational molecular testing would decipher intricate cases of chimeras.

In conclusion I would like to underline the conceptual value of the hypothesis for twin researches: 1) It places the curiosities of paternal-derived inherited twinning into the framework of current knowledge and verifiable genetic phenomena; 2) It predicts an existence of familial association of both twinning and infertility, called as Infertility/Twinning Paternally Dependent syndrome; infertility in these cases result in from such reproductive disorders as diandric triploidy and dipoid androgenesis (complete HD moles; 3) It predicts an occurrence in families with paternal-derived twinning of the third types of twins, sesquizygotic, intermediate between $\mathrm{MZ}$ and $\mathrm{DZ}$ twins; 4) It argued that families with paternal twinning may provide the valuable natural selective model for search of human chimeras and chimeric twins. The phenomenon of paternally-derived twinning seems now rather rare and marginal. However, the history of biology often proves the famous biblical maxim that the stone which the builders refused may become the head stone of the corner. In genetics the story of the discovery of mobile genes by Barbara McClintock is the most famous recent example.

\section{Acknowledgments}

The author is very grateful to Professors R. Derom and L. Keith for their interest to the study and encouragement, to Dr. G. Machin and Dr. K. McElreavey for discussions and comments and Dr. St Clair for critical reading of the manuscript and helpful remarks.

\section{References}

Andreano, G., Dobosz, M., Boschi, Liberti, A., \& Casa, D. (1998). Three sets of twins in three successive generations through the maternal line. Acta Geneticae Medicae et Gemellologiae (Roma), 47, 43-49.

Ardashnikov, S. N., Likhtenstein, E. A., Martynova, T. V., Soboleva, T. V., \& Postnikova, E. N. (1936). On diagnosing of zygosity of twins. Trudy Medical Genetics Institute (Russian), 4, 254-271.

Blickstein, I., \& Gurewitsch, E. D. (1998). Biblical twins. Obstetrics and Gynecology, 91, 632-634.

Boklage, C. E. (1987). Twinning, nonrighthandedness, and fusion malformations: Evidence for heritable causal elements held in common. American Journal of Medicine, 28, 67-84.

Boklage, C. E. (1995). The frequency and survival probability of natural twin conceptions. In L. G. Keith, E. Papiernik, D. M. Keith, \& B. Luke, Multiple Pregnancy (pp. 41-50). New York: Parthenon Publishing Group.

Bomsel-Helmreich, O., \& AlMufti, W. (1995). The mechanism of monozygosity and double ovulation. In L. G. Keith, E. Papiernik, D. M. Keith, \& B. Luke, Multiple pregnancy (pp. 25-40). New York: Parthenon Publishing Group.

Bulmer, M. G. (1970). The biology of twinning in man. Oxford: Clarendon Press.

Carmelli, D., Hasstedt, S., \& Anderson, S. (1981). Demography and genetics of human twinning in the Utah mormon genealogy. Progress in Clinical and Biological Research, 69A, 81-93. 
Chan, A. W. S., Dominko, T., Luetjens, C. M., Neuber, E., Martinovitch, C., Hewitson, L., Simerly, C. R., \& Schatten, G. P. (2000). Clonal propagation of primate offspring by embryo splitting. Science, 287, 317-319

Chitnis, S., Derom, C., Vlietinck, R., Derom, R., Monteiro, J., \& Gregersen, P. K. (1999). X-chromosome inactivation patterns confirm the late timing of monoamniotic-MZ twinning. American Journal of Human Genetics, 65, 570-571.

Dahlbom, M., Andersson, M., Lahdetie, J., Vierula, M., \& Alanko, M. (1997). Probable spermatozoal diploidy in the semen of golden retriever. Andrologia, 29, 49-55.

Davenport, C. B. (1920). Influence of the male in the production of human twins. American Nature, 54, 122.

Detwiler, S. (1933). Twinning in seven generations of the Bertolet family. Journal of Heredity, 24, 139-141.

Duffy, D. L., Montgomery, G. W., Hall, J., Mayne, C., Healey, S. C., Brown, J., Boomsma, D. I., \& Martin, N. G. (2001). Human twinning is not linked to the region of chromosome 4 synthetic with the sheep twinning gene FecB. American Journal of Medical Genetics, 100, 182-186.

Feng, Y. L., \& Gordon, J. W. (1996). Birth of normal mice after removal of the supernumerary male pronucleus from polyspermic zygotes. Human Reproduction, 11, 341-344.

Fitch, K., Yasuda, G., Owens, K. \& Wakimoto, B. (1998). Paternal effects in drosophila: Implications for mechanisms of early development. Current Topics in Developmental Biology, $38,1-34$.

Galloway, S. M., McNatty, K. P., Cambridge, L. M., Laitinen, M. P. E., Juengel, J. L., Jokiranta, T. S., McLaren, R. J., Luiro, K., Dodds, K. J., Montgomery, G. W., Beattie, A. E., Davis, G. H., \& Ritvos, O. (2000). Mutations in an oocyte- derived growth factor gene (BMP15) cause increased ovulation rate and infertility in a dosage-sensitive manner. Nature Genetics, 25, 279-283.

Gardner, R. L. (2001). The initial phase of embryonic patterning in mammals. International Review of Cytology, 203, 233-290.

Gedda, L., \& Brenci, G. (1986). Possible mechanism of the heredity of twinning. Acta Geneticae Medicae et Gemellologiae, 38, 3-5.

Gilbert, S. F. (1988). Developmental biology. Sunderland, MA: Sinauer Associates Inc.

Giltay, J. C., Brunt, T., Beemer, F. A., Wit, J. M., van Amstel, H. K., Pearson, P. L., \& Wijmenga, C. (1998). Polymorphic detection of a parthenogenetic maternal and double paternal contribution to a 46, XX/46,XY hermaphrodite. American Journal of Human Genetics, 62, 937-940.

Goldar, D. E., \& Kimberling, W. J. (1981). Genetic expectations of polar body twinning. Acta Geneticae Medicae et Gemellologiae, 30, 257-266.

Golubovsky, M. D. (1984). A pedigree in which multiple births are associated with infertility. Genetika (Russian), 20, 1052-1054.

Golubovsky, M. D. (1986). Genetics and Terah-Abraham pedigree in genesis. Koroth (Israel), 3(4), 374-382.

Golubovsky, M. D. (1998). Paternal genes and infertility/twinning paternally dependent syndrome. Twin Research, 1, 90.

Golubovsky, M. D., \& Golubovskaya, I. N. (1984). Possible cytogenetic mechanisms of direct paternal influence on twinning tendency in humans and their consequencies. Genetika, 20, 1052-1054.
Guttenbach, M., Martinez-Exposito, M. J., Michelmann, H. W., Engel, W., \& Schmid, M. (1997). Incidence of diploid and disomic sperm nuclei in 45 infertile men. Human Reproduction, 12, 468-473.

Haaf, T. (2001). The battle of the sexes after fertilization: behavior of paternal and maternal chromosome in the early mammalian embryo. Chromosome Research, 9, 263-271.

Hall, J. G. (1996). Twinning: Mechanisms and genetic implications. Current Opinions in Genetics and Development, 6, 343-347.

Harvey, M. A., Huntley, R. M., \& Smith, D. W. (1977). Familial monozygotic twinning. Journal of Pediatrics, 90, 246-248.

Hassold, T. J. (1986). Chromosome abnormalities in human reproductive wastage. Trends in Genetics, 4, 105-110.

Kanaev, I. I. (1959). Twins (in Russian; p. 382). Moscow - Leningrad: Izd Akad Nauk.

Keith, L., \& Machin, G. (1997). Zygosity testing: Current status and evolving issues. Journal of Reproductive Medicine, 42, 699-707.

Lichtenstein, P., Kallen, B., \& Koster, M. (1998). No paternal effect on monozygotic twinning in the Swedish twin registry. Twin Research, 1, 212-215.

Lindor, N. M., Ney, J., Jenkins, R., Thibodeau, S., Gordon, W., \& Dewald, W. A. A genetic review of complete and partial hydatidiform moles and nonmolar triploidy. Mayo Clinic Proceedings, 67, 791-799.

Machin, G. A. (1996). Some causes of genotypic discordance in monozygotic twin pairs. American Journal of Medical Genetics, 61, 216-228.

Machin, G. A., \& Bamforth, F. (1996). Zygosity and placental anatomy in 15 consecutive sets of spontaneously conceived triplets. American Journal of Medical Genetics, 61, 247-252.

Machin, G. A., \& Keith, L. G. (1999). An atlas of multiple pregnancy: Biology and pathology. The encyclopedia of visual medicine series. London: Parthenon Publishing Group.

McElreavey, K., \& Fellous, M. (1999). Sex determination and the Y chromosome. American Journal of Medical Genetics, 89, 176-185.

Meulemans, W. J., Lewis, C. M., Boomsma, D. I., Derom, C. A., Van den Berghe, H., Orlebeke, J. F., Vlietinck, R. F., \& Derom, R. M. (1996). Genetic modelling of dizygotic twinning in pedigrees of spontaneous dizygotic twins. American Journal of Medical Genetics, 61, 258-263.

Michels, V. V., \& Ricardi, V. M. (1981). Twin recurrence and amniocentesis: Male and MZ heritability factors. Birth Defects: Original Article Series, 14, 201-211.

Monteiro, J., Derom, C., Vlietinck, R., Kohn, N., Lesset, M., \& Gregersen, P. K. (1998). Commitment to X-inactivation precedes the twinning event in monochorionic MZ twins. American Human Genetics, 6, 339-346.

Moglabey, Y. B., Kircheisen, R., Seoud, M., Mogharbel, N. E., Van den Veyer, I., \& Slim, R. (1999). Genetic mapping of a maternal locus responsible for familial hydatidiform moles. Human Molecular Genetics, 8, 667-671.

Ogura, A., Suzuki, O., Tanemura, K., Mochida, K., Kobayashi, Y., \& Matsuda, J. (1998). Development of normal mice from methaphase I oocytes fertilized with primary oocytes. Proceedings of the National Academy of Sciences of the USA, 95, 561-565. 
Ogura, A., Wakayama, T., Suzuki, O., Shin, T. Y., Matsuda, J., \& Kobayashi, Y. (1997). Chromosome of mouse primary spermatocytes undergo meiotioc divisions after incorporation into homologous immature oocytes. Zygote, 5, 177-182.

Oster, H. (2001). Sex determination: Lessons from families and embryos. Clinical Genetics, 59, 207-215.

Pal, L., Toth, T. L., Leykin, L., \& Isaakson, K. B. (1996). High incidence triploidy in vitro fertilized oocytes from a patient with a previous history of a recurrent gestational trophoblastic disease. Human Reproduction, 11, 1529-1532.

Palermo, G., Munne, S., \& Cohen, J. (1994). The human zygote inherits its mitotic potential from the male gamete. Human Reproduction, 9, 1220-1225.

Palermo, G. D., Munne, S., Colombero, L. T., Cohen, J., \& Rosenwaks, Z. (1995). Genetics of abnormal human fertilization. Human Reproduction, 10(Suppl 1), 120-127.

Parisi, P., Gatti, M., Prinzi, G., \& Caperna, G. (1983). Familial incidence of twinning. Nature, 304, 626-628.

Pruett, L. R., \& Zinn, A. R. (2001). A fork in the road to fertility. Nature Genetics, 27, 132-134.

Robson, P., Stein, P., Xhou, B., Schultz, R., \& Baldwin, H. C. (2001). Inner cell mass-specific expression of a cell adhesion molecule (Pecam-1/CD31) in the mouse blastocyst. Developmental Biology, 234, 317-329.

Sasagawa, I., Kuretake, S., Eppig, J. J., \& Yanagimachi, R. (1998). Mouse primary oocytes can complete two meiotic divisions within the oocyte cytoplasm. Biology of Reproduction, 58, 248-254.

Sathananthan, A. H. (1998). Paternal centrosomic dynamics in the early human development and infertility. Journal of Assisted Reproduction and Genetics, 15, 129-138.

Shapiro, L. R., Zemek, L., \& Shulman, N. (1981). Genetic etiology of monozygotic twinning. Birth Defects: Original Article Series, 14, 219-222.

Sills, S. E., Moomjy, M., Zaninovic, M., Veeck, L., McGee, M., Palermo, G. D., \& Rozenwaks, Z. (2000). Human zona pellucida micromanipulation and monozygotic twinning frequency after IVF. Human Reproduction, 15, 890-895.

St Clair, D., St Clair, J., Swainson, C. H., Bamforth, F., \& Machin, G. (1998). Twin zygosity testing for medical purposes. American Journal of Medical Genetics, 77, 412-414.

St Clair, J., \& Golubovsky, M. (2002). Paternally derived twinning: A two century examination of records of one Scottish name. Twin Research, (submitted).
Strain, L., Dean, J., Hamilton, M., \& Bonthron, D. T. (1998). A true hermaphrodite chimera resulting from embryo amalgamation after in vitro fertilization. New England Journal of Medicine, 338, 166-169.

Strain, L., Warner, J. P., Johnston, T., \& Bonthron, D. (1995). A human parthenogenetic chimera. Nature Genetics, 11, 164-169.

Surani, M. (1995). Parthenogenesis in man. Nature Genetics, $11,111-113$.

Sutovsky, P., \& Schatten, G. (2000). Paternal contributions to the mammalian zygote: Fertilization after sperm-egg fusion. International Review of Cytology, 195, 61-65.

Taylor, S. A., \& Braude, P. R. (1994). The early development and DNA content of activated uman oocyters and partenogenetic human embryos. Human Reproduction, 9, 2389-2397.

Uchida, I. A., \& Freeman, V. C. (1985). Triploidy and chromosomes. American Journal of Obstetrics and Gynecology, $151,65-69$.

Uehara, S., Nata, M., \& Nagae, M. (1995). Molecular biological analyses of tetragametic chimerism in a true hermaphrodite with 46, XX/46 XY. Fertility and Sterility, 63, 189-192.

Verp, M. S., Harrison, H. H., Ober, C., Oliveri, D., Amarose, A., Lindgren, V., \& Talerman, A. (1992). Chimerism as etiology of a 46, XX/46, XY fertile true hermaphrodite. Fertility and Sterility, 57, 345-349.

Warburton, D. (1997). Human female meiosis: New insight into an error-prone process. American Journal of Human Genetics, $61,1-4$.

Wasserman, P. M., Jovine, L., \& Litscher, E. (2001). A profile of fertilization in mammals. Nature cell biology, 3, 59-64.

Zaragoza, M., Surti, U., Redline, R. W., Millie, E., Chakravarti, A., \& Hassold, T. (2000). Parental origin and phenotype of triploidy in spontaneous abortions: Predominance of diandry and association with partial hydatidiform mole. American Journal of Human Genetics, 66, 1807-1820.

Zuelzer, W. W., Beattie, K. M., \& Reisman, L. E. (1964). Generalized unbalanced mosaicism attributable to dispermy and probable fertilization of a polar body. Human Genetics, $16,39-51$.

Zenzes, M. T., \& Casper, R. F. (1992). Cytogenetics of human oocytes, zygotes and embryos after in vitro fertilization. Human Genetics, 88, 367-375. 